\title{
A New Iterational Method for Ordinary Equations Using Sumudu Transform
}

\author{
Yanqin $\mathrm{Liu}^{1}, 2^{*}$ and Wen Chen ${ }^{1}$ \\ ${ }^{1}$ Department of Engineering Mechanics, Hohai University, Jiangsu, 210098, China \\ ${ }^{2}$ School of Mathematical Sciences, Dezhou University, Dezhou 253023, China \\ Email: yanqinliu@dzu.edu.cn
}

\begin{abstract}
A novel modification of the variational iteration method (VIM) is proposed by means of the Sumudu transform. This approach is a universal way to identify the multiplier, which is simple and effective, and successfully extended to ordinary differential equations, including the nonlinear and variable coefficient ones. The Lagrange multiplier can be easily identified by the Sumudu transform and the nonlinear term will be handled by the Adomian series. Examples are given to elucidate the process and the reliability of the method.
\end{abstract}

Keywords: Approximate solution, variational iteration method, Lagrange multiplier, Sumudu transform, nonlinear equation.

\section{Introduction}

The variational iteration method (VIM), was first proposed and systematically elucidated by He[1,2,3]. This approach is an analytical technique which has been widely used in the past ten years[4,5,6,7], and its results in approximate solutions have high accuracies. With the widely application of this method, many researchers make efforts to its developments $[8,9,10,11]$. Notably, $\mathrm{Wu}[12,13,14,15]$ used Laplace transform to identify the Lagrange multipliers, which overcomes principle drawbacks in application of the VIM to fractional equations.

Recently, Sumudu transform is adopted in some famous analytical methods $[16,17,18]$, the combination of Sumudu transform and homotopy perturbation method is used to simplify the solution process and improve the solution's accuracy.

Motivated and inspired by Wu's[12,13] thinking, and combining with the Sumudu transform, we give a new modified variational iteration method, which is based on variational iteration theory and Sumudu transform. In this work, we will use this method to solve some ordinary equations, including the nonlinear and variable coefficient differential equations. The Lagrange multiplier can be easily identified by the Sumudu transform and the nonlinear term will be handled by the Adomian series. The results show that this new modification has high accuracy and simplicity.

\section{New Identification of the Lagrange Multipliers}

\subsection{Fundamental Facts of the Sumudu Transform}

The Sumudu transform is an integral transform, which was first introduced by Watugala[19] to solve differential equations and control engineering problems.

Definition 1 The Sumudu transform of a function $f(t)$, defined for all real numbers $t \geq 0$, is the function $F(u)$, defined by[19]

$$
F(u)=S[f(t)]=\int_{o}^{\infty} f(u t) e^{-t} \mathrm{~d} t .
$$

Proposition 1 The Sumudu transform of the derivatives with integer order is given as follows[19]

$$
S\left[\frac{\mathrm{d} f(t)}{\mathrm{d} t}\right]=\frac{1}{u}[F(u)-f(0)] .
$$


we can also get the Sumudu transform of the $n$-order derivative as follows:

$$
S\left[\frac{\mathrm{d}^{n} f(t)}{\mathrm{d} t^{n}}\right]=\frac{1}{u^{n}}\left[F(u)-\left.\sum_{k=0}^{n-1} u^{k} \frac{\mathrm{d}^{k} f(t)}{\mathrm{d} t^{k}}\right|_{t=0}\right] .
$$

\subsection{Description of the New Modified Method}

In order to elucidate the procedure of this approach, we consider the following general nonlinear system:

$$
\begin{gathered}
\frac{\mathrm{d}^{n} w(t)}{\mathrm{d} t^{n}}+R[w(t)]+N[w(t)]=g(t), \\
w^{(k)}(0)=\frac{\mathrm{d}^{k} w(0)}{\mathrm{d} t^{k}}=a_{k},
\end{gathered}
$$

where $k=0, \cdots, n-1, g(t)$ is the source term, $N$ represents the nonlinear operator and $R$ is the linear operator. $\frac{\mathrm{d}^{n} w(t)}{\mathrm{d} t^{n}}$ is the term of the highest-order derivative.

Inspired by Wu's[12,13] method, if we take Sumudu transform on both sides of Eqs.(4-5), the linear part with constant coefficients is then transferred into an algebraic one, so that we can identify the Lagrange multiplier in a more straightforward way.

Now, we extend this idea to find the unknown Lagrange multiplier. Taking the above Sumudu transform to both sides of Eq.(3) and (4), then the linear part is transformed into an algebraic equation as follows:

$$
\frac{1}{u^{m}} W(u)-\sum_{k=0}^{m-1} \frac{1}{u^{m-k}} w^{(k)}(0)+S[R[w]]+S[N[w]]-S[g(t)]=0,
$$

where $W(u)=S[w(t)]$. The iteration formula of (6) can be used to suggest the main iterative scheme involving the Lagrange multiplier as

$$
\begin{gathered}
W_{n+1}(u)=W_{n}(u)+\lambda(u)\left[\frac{1}{u^{m}} W_{n}(u)-\sum_{k=0}^{m-1} \frac{1}{u^{m-k}} w^{(k)}\left(0^{+}\right)\right. \\
+S[R[w]]+S[N[w]]-S[g(t)]],
\end{gathered}
$$

Considering $S[R[w]]+S[N[w]]$ as restricted terms, and taking the classical variation operator on both sides of $(7)$

$$
\delta W_{n+1}(u)=\delta W_{n}(u)+\lambda(u) \frac{1}{u^{m}} \delta W_{n}(u),
$$

one can derive a Lagrange multiplier as

$$
\lambda=-u^{m},
$$

With Eq.(9) and the inverse-Sumudu transform $S^{-1}$, the iteration formula (7) can be explicitly given as

$$
\begin{gathered}
w_{n+1}(t)=w_{n}(t)+S^{-1}\left[-u^{m}\left[\frac{1}{u^{m}} W_{n}(u)-\sum_{k=0}^{m-1} \frac{1}{u^{m-k}} w^{(k)}\left(0^{+}\right)+S\left[R\left[w_{n}\right]\right]+S\left[N\left[w_{n}\right]\right]-S[g(t)]\right]\right] \\
=w_{0}(t)+S^{-1}\left[-u^{m}\left[S\left[R\left[w_{n}(t)\right]+N\left[w_{n}(t)\right]\right]\right],\right.
\end{gathered}
$$

where $w_{0}(t)$ is an initial approximation of Eq.(4), and

$$
\begin{gathered}
w_{0}(t)=S^{-1}\left(\sum_{k=0}^{m-1} u^{k} w^{(k)}(0)\right)+S^{-1}\left[u^{m} S[g(t)]\right] \\
=w(0)+w^{\prime}(0) t+\cdots+\frac{w^{m-1}(x, 0) t^{m-1}}{(m-1) !}+S^{-1}\left[u^{m} S[g(t)]\right] .
\end{gathered}
$$




\section{$3 \quad$ Illustrative Examples}

\subsection{Linear and Nonlinear ODEs}

Example 1 Let us consider the following simple linear differential equation:

$$
\frac{\mathrm{d} w(t)}{\mathrm{d} t}=a w(t), \quad w(0)=w_{0}
$$

which has the exact solution $w(t)=w_{0} e^{a t}$. Using the procedure in Section 2, we can obtain the successive formula

$$
\left\{\begin{array}{l}
w_{0}(t)=w(0)=w_{0} \\
w_{n+1}(t)=S^{-1}\left[w_{0}+u S\left[a w_{n}(t)\right]\right]
\end{array}\right.
$$

and the approximate solution

$$
\left\{\begin{aligned}
w_{0}(t)= & w_{0} \\
w_{1}(t)= & w_{0}+S^{-1}\left[u S\left[a w_{0}(t)\right]\right]=w_{0}+w_{0} a t \\
w_{2}(t)= & w_{0}+S^{-1}\left[u S\left[a w_{1}(t)\right]\right]=w_{0}+w_{0} a t+w_{0} \frac{a^{2} t^{2}}{2 !} \\
& \vdots \\
w_{n}(t)= & w_{0}+S^{-1}\left[u S\left[a w_{n-1}(t)\right]\right]=w_{0}\left(1+a t+\frac{a^{2} t^{2}}{2 !}+\cdots\right)
\end{aligned}\right.
$$

for $n \rightarrow \infty, w_{n}(t)$ tends to the exact solution $w_{0} e^{a t}$.

Example 2 Let us consider the following nonlinear logistic equation[5]:

$$
\frac{\mathrm{d} w(t)}{\mathrm{d} t}=\mu w(t)(1-w(t)), \quad w(0)=\frac{1}{2} .
$$

Taking the above procedure, and the iteration formula of (15) involving the Lagrange multiplier $\lambda(u)=-u$ is given

$$
w_{n+1}(t)=w_{0}(t)+S^{-1}\left[\mu u\left[S\left[w_{n}\right]-S\left[N\left[w_{n}\right]\right]\right]\right]
$$

$w_{0}(t)=\frac{1}{2}$ is an initial approximation of Eq.(15). Let $w_{n}=\sum_{i=0}^{n} v_{i}$, and nonlinear term can be decomposed as $N[w]=\Sigma_{i=0}^{\infty} A_{i}, A_{i}=\left.\frac{1}{i !}\left[\frac{d^{i}}{d \lambda^{2}} \Phi\left(\sum_{n=0}^{\infty} \lambda^{n} u_{n}\right)\right]\right|_{\lambda=0}$, where $A_{n}$ is the Adomian polynomials[20], then the Adomian series of the term $v^{2}$ reads

$$
\left\{\begin{array}{l}
A_{0}=v_{0}^{2}, \\
A_{1}=2 v_{0} v_{1}, \\
A_{2}=2 v_{0} v_{2}+v_{1}^{2}, \\
\quad \vdots
\end{array}\right.
$$

so we can derive the following iteration formula

$$
\left\{\begin{array}{l}
v_{n+1}(t)=S^{-1}\left[\mu u\left[S\left[v_{n}\right]-S\left[A_{n}\right]\right]\right] \\
v_{0}=w_{0}
\end{array}\right.
$$

with symbolic computation, we can derive the following approximate solutions

$$
\left\{\begin{array}{l}
v_{0}=\frac{1}{2} \\
v_{1}=\frac{\mu t}{4} \\
v_{2}=0 \\
v_{3}=-\frac{\mu^{3} t^{3}}{48} \\
\quad \vdots
\end{array}\right.
$$

so the exact solution is given by

$$
w(t)=\lim _{n \rightarrow \infty} w_{n}=\lim _{n \rightarrow \infty} \sum_{i=0}^{n} v_{i}==\frac{1}{2}+\frac{\mu t}{4}-\frac{\mu^{3} t^{3}}{48}+\frac{\mu^{5} t^{5}}{480}+\cdots
$$

This in turn gives the exact solution $[5] w(t)=\frac{e^{\mu t}}{1+e^{\mu t}}$. 


\subsection{Variable Coefficient Ordinary Equation}

Now consider the system (4) with variable coefficients and assume the linear term as

$$
R[w(t)]=a R_{1}[w(t)]+b(t) R_{2}[w(t)]
$$

where $R_{1}, R_{2}$ are also the linear operator, $a$ is a constant and $b(t)$ is a variable coefficient, then the new modified VIM is given as follows: Now, with Eq.(21) and taking the above Sumudu transform to both sides of Eq.(4) and (5), then the iteration formula becomes

$$
\begin{gathered}
W_{n+1}(u)=W_{n}(u)+\lambda(u)\left[\frac{1}{u^{m}} W_{n}(u)-\sum_{k=0}^{m-1} \frac{1}{u^{m-k}} w^{(k)}\left(0^{+}\right)\right. \\
\left.+S\left[a R_{1}[w(t)]\right]+S\left[b(t) R_{2}[w(t)]\right]+S[N[w]]-S[g(t)]\right],
\end{gathered}
$$

Considering $S\left[b(t) R_{2}[w(t)]\right]+S[N[w]]$ as restricted terms, the Lagrange multiplier will be easily obtain, then repeat the above iteration process.

Example 3 Consider the following differential equations with a variable coefficient as an example

$$
\frac{\mathrm{d} w(t)}{\mathrm{d} t}-(1+2 t) w(t)=0, \quad w(0)=1
$$

Taking Sumudu transform on both sides of Eq.(23)

$$
\frac{W(u)}{u}-\frac{1}{u}-W(u)-S[2 t w(t)]=0
$$

construct the variational iteration formula

$$
W_{n+1}(u)=W_{n}(u)+\lambda(u)\left[\frac{1}{u} W_{n}(u)-\frac{1}{u}-W_{n}(u)-S\left[2 t w_{n}(t)\right]\right],
$$

Considering the term $\mathrm{S}[2 \mathrm{tw}(\mathrm{t})]$ is restricted variations, take the classical variation operator on both sides of $(25)$

$$
\delta W_{n+1}(u)=\delta W_{n}(u)+\lambda(u)\left(\frac{1}{u}-1\right) \delta W_{n}(u)
$$

a Lagrange multiplier can be determined as $\lambda(u)=\frac{u}{u-1}$. Taking inverse-Sumudu transform $S^{-1}$, the iteration formula (25) can be explicitly given as

$$
\begin{aligned}
w_{n+1}(t)=w_{n}(t) & +S^{-1}\left[\frac{u}{u-1}\left[\frac{1}{u} W_{n}(u)-\frac{1}{u}-W_{n}(u)-S\left[2 t w_{n}(t)\right]\right]\right. \\
& =w_{0}(t)-S^{-1}\left[\frac{u}{u-1} S\left[2 t w_{n}(t)\right]\right]
\end{aligned}
$$

where $w_{0}(t)$ is an initial approximation of Eq.(23), and

$$
w_{0}(t)=S^{-1}\left[\frac{1}{1-u}\right]=e^{t}
$$

after a series of computation, we can derive the following approximate solutions

$$
\left\{\begin{array}{l}
w_{1}(t)=e^{t}\left(1+t^{2}\right) \\
w_{2}(t)=e^{t}\left(1+t^{2}+\frac{t^{4}}{2}\right) \\
w_{3}(t)=e^{t}\left(1+t^{2}+\frac{t^{4}}{2}+\frac{t^{6}}{6}\right) \\
\vdots
\end{array}\right.
$$

for $n \rightarrow \infty, w_{n}(t)$ tends to the exact solution $e^{t+t^{2}}$. 


\section{Conclusion}

A new approach based on the Sumudu transform is proposed to identify the Lagrange multipliers of the VIM. With this new method, we can easily derive Lagrange multipliers without tedious calculation and the new variational iteration formula can be derived. Results show that this method is powerful to solving ordinary differential equations, even for nonlinear and variable coefficient equations.

This new modification here transfers the problem into the algebraic equation in the Sumudu $u$-domain, this idea can also be extended to the fractional calculus of variations, and the Lagrange multipliers can be determined readily from Sumudu transform, and our successive research will be conducted on the fractional equations.

Acknowledgments. We express our thanks to the referees for their fruitful advices and comments. And this article is supported by National Natural Science Foundation of China (No. 11501082), Natural Science joint special Foundation of Shandong Province (No. ZR2015AL016) and Postdoctoral Science Foundation of Jiangsu Province(Grant No.1402046C.)

\section{References}

\section{References}

1. J.H. He, "Variational iteration method-a kind of nonlinear analytical technique: some examples", International Journal of Non-Linear Mechanics, Vol. 34 No. 4, pp. 699-708,1999.

2. J.H. He, X.H. Wu, "Variational iteration method: new development and applications", Computers and Mathematics with Applications, Vol. 54 No.7-8, pp.881-894, 2007.

3. J.H. He, "Asympototic methods for solitary solutions and compactons",Abstract and Applied Analysis, Vol. 2012, Article ID 916793, 2012.

4. A.M. Wazwaz, "A comparison between the variational iteration method and Adomian decomposition method", Journal of Computational and Applied Mathematics, Vol. 207 No. 1, pp. 129-136, 2007.

5. A.M. Wazwaz, "The variational iteration method for analytic treatment for linear and nonlinear ODEs", Applied Mathematics and Coputation, Vol. 212 No. 1, pp. 120-134, 2009.

6. M.A. Noor, S.T. Mohyud-Din, "Variational iteration method for solving higher-order nonlinear boundary value problems using He's polynomials", , Vol. 9 No. 2, pp. 141-156, 2008.

7. M. A. Noor, "Some iterative methods for solving nonlinear equations using homotopy perturbation method", International Journal of Computer Mathematics", Vol. 87 No. 1, pp. 141-149, 2010.

8. F. Geng, "A modified variational iteration method for solving Riccati differential equations", Computers and Mathematics with Applications, Vol. 60 No. 7, pp. 1868-1872, 2010.

9. H. Jafari, C.M. Khalique, "Homotopy perturbation and variational iteration methods for solving fuzzy differential equations", Communications in Fractional Calculus, Vol. 3 No. 1, pp. 38-48, 2012.

10. Y.Q. Liu, "Variational homotopy perturbation method for solving fractional initial boundary value problems", Abstract and Applied Analysis, Vol. 2012, Article ID 727031, 10 pages, 2012.

11. Y.Q. Liu, F.S. Xu, X.L. Yin, "Variational approximate solutions of fractional nonlinear nonhomogeneous equations with laplace transform", Abstract and Applied Analysis,Vol. 2013, Article ID 819268, 9 pages, 2013.

12. G.C. Wu, "Challenge in the variational iteration method - A new approach to identification of the lagrange multipliers", Journal of King Saud University-Science, Vol. 25 No. 2, pp. 175-178, 2013.

13. G.C. Wu, "Laplace transform overcoming principle drawbacks in application of the variational iteration method to fractional heat equations", Thermal Science, Vol. 16 No. 4, pp. 1257-1261, 2012.

14. G.C. Wu, D. Baleanu, "Variational iteration method for fractional calculus - a universal approach by laplace transform", Advances in Difference Equations, 2013: 18 pp. 1-9, 2013.

15. G.C. Wu, D. Baleanu, "Variational iteration method for the Burgers' flow with fractional derivatives- New Lagrange multipliers", Applied Mathematical Modelling, Vol. 37 No. 9, pp. 6183-6190, 2013.

16. Abdon Atangana, Necdet Bildik, and S.C. Oukouomi Noutchie, "New iteration methods for time-fractional modified nonlinear kawahara equation", Abstract and Applied Analysis, Vol. 2014, Article ID 740248, 9 pages, 2014 .

17. Hasan Bulut, Haci Mehmet Baskonus, and Tethi Bin Muhammad Belgacem, "The analytical solution of some fractional ordinary differential equations by the sumudu transform method", Abstract and Applied Analysis, Vol. 2013, Article ID 203875, 6 pages, 2013. 
18. A.A. Elbeleze, A. kiliçman, B.M. Taib, " Homotopy perturbation method for fractional Block-Scholes european option pricing equations using Sumudu transform", Mathematical Problems in Engineering, Vol. 2013, Article ID 524852, 7 pages, 2013.

19. G.K. Watugala, "Sumudu transform-a new integral transform to solve differential equations and control engineering problems", Mathematical Engineering in Industry, Vol. 24 No. 1, pp. 35-43, 1993.

20. G. Adomian, "A review of the decomposition method in applied mathematics", Journal of Mathematical Analysis and Applications, Vol. 135 No. 2, pp. 501-544, 1988. 